\title{
A Using Electromagnetic Signal Propagation Models for Radio and Television Broadcasts: An Introduction
}

\author{
Charles Crabtree' and Holger L. Kern ${ }^{2}$
}

\author{
${ }_{1}^{1}$ PhD cand., Department of Political Science, University of Michigan, Ann Arbor, MI 48109, USA. Email: ccrabtr@umich.edu \\ ${ }^{2}$ Assistant Professor, Department of Political Science, Florida State University, Tallahassee, FL 32306, USA. \\ Email:hkern@fsu.edu
}

\begin{abstract}
This note offers an introduction to electromagnetic signal propagation models, which can be used to model terrestrial radio and television signal strength across space. Such data are useful to social scientists interested in identifying the effects of mass media broadcasts when (i) individual-level data on media exposure do not exist or when (ii) media exposure, while observed, is not exogenous. We illustrate the use of electromagnetic signal propagation models by creating a signal strength measure of military-controlled radio stations during the 2012 coup in Mali.
\end{abstract}

Keywords: radio, television, signal propagation, Natural experiments, Longley-Rice

\section{Introduction}

A large and well-developed literature examines the role that radio and television play in shaping a wide variety of political behaviors from voting to conflict. However, this research is confronted with significant data availability and causal identification problems. First, it is frequently difficult to obtain individual-level data on media exposure. This is particularly true in non-Western or autocratic contexts where reliable survey data often do not exist. The lack of survey data also poses a barrier to studies of media effects in historical settings. Second, identifying the causal effects of media exposure is complicated by the endogeneity of both media availability and consumption.

In this paper, we provide an accessible introduction to an approach that, under conditions we discuss below, can be used to address both of these issues. Generally, terrestrial radio and television signal strength-and thus radio and television availability-varies as a function of transmitter characteristics and topography. This variation in signal strength, which is often plausibly exogenous (at least conditional on observables), can be used as a natural source of causal identification. Examples of this identification strategy include work on the effects of radio and television consumption on social capital in Indonesia (Olken 2009), the link between hate radio and collective violence in Rwanda (Yanagizawa-Drott 2014), radio's impact on the rise of the Nazis (Adena et al. 2015), West German television's role during the East German revolution (Crabtree, Darmofal, and Kern 2015), the effects of Russian television on voting behavior in Ukraine (Peisakhin and Rozenas 2017), and the effects of entertainment television in Italy on votes for populist parties (Durante, Pinotti, and Tesei 2017).

Despite this recent flurry of work employing electromagnetic signal propagation models, we lack an introduction to these models geared toward social scientists. So far, social scientists

Charles Crabtree

Edited by

Justin Grimmer

(c) The Author(s) 2018. Published by Cambridge University Press on behalf of the Society for Political Methodology. 
Table 1. Radio signals.

\begin{tabular}{|c|c|c|c|}
\hline Band & Frequency & Primary means of propagation & Example technologies \\
\hline Low Frequency (LF) & $30-300 \mathrm{kHz}$ & ground wave and skywave & AM radio \\
\hline $\begin{array}{l}\text { Medium Frequency } \\
\text { (MF/Short wave) }\end{array}$ & $300-3000 \mathrm{kHz}$ & ground wave and skywave & AM radio \\
\hline $\begin{array}{l}\text { High Frequency } \\
\text { (HF/Short wave) }\end{array}$ & $3-30 \mathrm{MHz}$ & skywave & $\begin{array}{l}\text { International radio, } \\
\text { weather stations }\end{array}$ \\
\hline $\begin{array}{l}\text { Very-high Frequency } \\
\text { (VHF) }\end{array}$ & $30-300 \mathrm{MHz}$ & line of sight & FM radio, television \\
\hline $\begin{array}{l}\text { Ultrahigh Frequency } \\
\text { (UHF) }\end{array}$ & $300-3000 \mathrm{MHz}$ & line of sight & Wi-Fi, bluetooth \\
\hline $\begin{array}{l}\text { Super-high Frequency } \\
\text { (SHF) }\end{array}$ & $3-30 \mathrm{GHz}$ & line of sight & Cell phones \\
\hline
\end{tabular}

Source: Seybold (2005).

interested in using these models to exploit variation in radio or television signal strength have been forced to synthesize highly technical information from engineering literatures that presuppose considerable technical knowledge. The goal of this note is to provide a stand-alone, applied guide to signal propagation models. In the next section, we provide a brief primer on radio waves and signal propagation and introduce two of the models commonly used to model signal strength. We then discuss how these models can be implemented and highlight best practices. Next, we describe the model output and how to best incorporate it into empirical analyses followed by a discussion of causal identification strategies. We conclude with a brief empirical example.

\section{Radio Waves and Modeling Radio Wave Propagation}

Radio waves can propagate in three ways. First, they can travel via line of sight (direct wave): if individuals can see the transmitter they can receive its signal. Second, they can travel as ground waves following the curvature of the Earth. Transmissions sent this way can be received when the transmitter has dropped below the horizon or when topographic barriers prevent line-of-sight reception. Third, radio waves can travel via skywave. Waves are transmitted upward, reflect off charged particle layers in the ionosphere, and then return to Earth. Skywave propagation typically occurs over much greater distances than ground wave propagation.

Different types of radio waves travel via different means. This is important because signal propagation models are designed to model certain types of signals. Table 1 classifies the most commonly used radio waves by band, lists the range of frequencies in each band, notes their primary means of propagation, and provides examples of technologies transmitting on frequencies within these bands. Low-, medium-, and high-frequency bands are used to transmit radio broadcasts, very-high-frequency bands are used to transmit radio and television broadcasts, and super-high-frequency bands are used to transmit cell phone signals. Waves in the lowand medium-frequency bands can travel via both ground wave and skywave; waves in the high-frequency band primarily travel through the sky; waves in the very-high-frequency and super-high-frequency bands primarily travel via line of sight.

\subsection{Modeling electromagnetic signal propagation}

There exists a dizzying range of electromagnetic signal propagation models. Many have very specific uses and can largely be ignored by social scientists. We focus here on two workhorse models-the Irregular Terrain Model (ITM), also known as the Longley-Rice Model, and the Terrain Integrated Rough Earth Model (TIREM). From the point of view of the applied researcher, these 
models are similar in many important ways. In general terms, they both model electromagnetic signals over a predefined geographic area. Each generates a measure of electromagnetic signal strength at one or more points in space.

Model predictions have been repeatedly validated via on-the-ground measurements (Longley and Rice 1968; Eppink and Kuebler 1994; Seybold 2005; Lazaridis et al. 2013). Due to the high accuracy of these models, they are commonly employed to calculate signal strength for commercial and defense applications. Since its development in 1968, ITM has been regularly used by the United States government, nongovernmental organizations, and businesses to model signal propagation. It is the model most commonly used by the U.S. Federal Communications Commission (DeBolt n.d.; Longley and Rice 1968; Hufford, Longley, and Kissick 1982; National Institute of Standards \& Technology 2004, 36; Seybold 2005, 143). TIREM started as a minor derivative of ITM but has been modified over time to account for some of ITM's weaknesses. It is the standard model used by United States defense agencies (Jones, Layer, and Osenkowsky 2013). Social science researchers have used both of these models (e.g., Olken 2009; Enikolopov, Petrova, and Zhuravskaya 2011; DellaVigna et al. 2014; Yanagizawa-Drott 2014; Adena et al. 2015; Crabtree et al. 2015; Durante et al. 2017; Peisakhin and Rozenas 2017; Crabtree, Kern, and Pfaff forthcoming).

ITM is designed to model transmissions in the $20 \mathrm{MHz}$ to $20 \mathrm{GHz}$ range (Hufford et al. 1982). TIREM is designed to cover the range from $1 \mathrm{MHz}$ to $40 \mathrm{GHz}$ (Eppink and Kuebler 1994). ${ }^{1}$ ITM and TIREM generally offer equally accurate predictions in the $20 \mathrm{MHz}$ to $20 \mathrm{GHz}$ range, although ITM is thought to be slightly more accurate for modeling VHF transmissions (Seybold 2005). TIREM is strictly preferred when modeling signal propagation over large bodies of water. In practice, ITM is more commonly used than TIREM because it is better documented, easier to modify, and more widely implemented in software (Jones, Layer, and Osenkowsky 2013).

Model predictions are based on three sets of inputs. The first set includes the location of the broadcast transmitter and its technical characteristics, such as antenna height, power, and frequency (Eppink and Kuebler 1994). These are the primary factors influencing signal propagation. Other factors related to a transmitter's location, such as climate zone type and ground conductivity, can influence the strength of the signal near its origin, thereby affecting signal strength downstream (DeBolt n.d.). The second set includes topographic data, which are used to account for the fact that hilly or mountainous terrain can strongly influence signal strength by refracting or diffracting radio waves. The third set includes receiver characteristics such as reception location and antenna height.

\section{Using ITM and TIREM}

ITM and TIREM can be implemented in many software packages. We list all options that we are aware of in the online appendix. Since these programs tend to offer similar sets of features, we keep our discussion of implementation general and focus on the data needs and modeling decisions that researchers face.

At a minimum, the following transmitter characteristics are needed: location (latitude and longitude), antenna height, effective radiated power (ERP, measured in kW), and broadcast frequency. ${ }^{2}$ Sufficient transmitter data can typically be collected through published, official, or archival sources. Many websites run by radio enthusiasts also provide detailed information about radio and television transmitters worldwide. ${ }^{3}$

1 Both models are commonly used outside these ranges, which results in somewhat diminished accuracy. If researchers are primarily interested in modeling signals in the low-frequency band they should use the VOACAP model available at http://www.voacap.com/.

2 Incorporating additional transmitter information such as modulation, polarization, or broadcasting direction will improve precision. If unavailable, software generally uses plausible defaults.

3 Examples include www.fmlist.org and www.mwlist.org. 
Global terrain data are also needed but often supplied with the modeling software. They can also easily be found online. ${ }^{4}$ While ITM and TIREM can incorporate precise information about radio and television receivers, it is rarely the case that researchers possess sufficiently detailed information. As a result, it has become standard practice to leave receiver characteristics at their default values, with one exception; when the height of the receiver antenna is unknown, it is commonly set to 10 meters above ground (DeBolt n.d.).

Signal propagation modeling requires a number of additional decisions. First, software typically offers the option to either generate a coverage map for a specific area or to calculate signal strength for a particular transmitter-receiver pair (point-to-point). If the number of locations for which signal strength information is required is relatively small, point-to-point modeling is preferred since it is the most accurate. Area coverage maps are slightly less precise but more feasible computationally when signal strength measurements are needed for a large area or many specific locations. For example, Crabtree et al. (2015) use an area coverage map of East Germany to approximate the signal strength of West German television transmitters across East German counties during the East German revolution.

Second, if a broadcaster uses multiple transmitters researchers need to decide how to combine their signal strengths. For each transmitter, ITM or TIREM will calculate a coverage map (in area mode) or signal strength at specific locations (in point-to-point mode). The standard approach for combining these transmitter-specific predictions is to use the maximum signal strength in any given area or location (e.g., Enikolopov et al. 2011, 3264). Peisakhin and Rozenas (2017) exploit survey information about Russian television reception across the Ukrainian precincts in their sample to choose the optimal way of combining signal strengths from several transmitters. In our view, incorporating such external information is valuable because it provides an empirical benchmark. At the same time, in our experience it often yields results that are not very different from using the maximum signal strength across transmitters.

Third, researchers need to choose the units of measurement for signal strength. The typical unit for measuring received signal from radio or television broadcasts is $\mathrm{dB} \mu \mathrm{V} / \mathrm{m}$, or electric field strength relative to 1 microvolt per meter (Seybold 2005). Higher values represent better signal (see below).

\subsection{Model output}

In area mode, software will output a raster image that displays signal strength at the $1 \mathrm{~km} \times 1 \mathrm{~km}$ level. GIS software can be used to aggregate these signal strength data to an appropriate level of analysis (e.g., county or municipality). In point-to-point mode, modeling software will provide signal strengths for the selected locations.

Signal strength measures can be incorporated into empirical analyses in several different ways. The first approach simply uses the untransformed measure as a continuous treatment or instrumental variable. When doing so it will be important to allow for a flexible functional form between signal strength and the dependent variable. While one would expect the relationship to be weakly monotonic, it is unlikely to be linear. ${ }^{5}$

The second approach explicitly models the relationship between signal strength and media availability. Enikolopov et al. (2011), for example, regress an indicator for television availability from survey data on signal strength and then use the predicted probability of television availability across Russian subregions as treatment variable. The main advantage of this approach is that it allows for an easier interpretation of effect sizes. It also addresses the fact noted above that only

4 http://www.ve2dbe.com/dataen.html or http://dds.cr.usgs.gov/srtm/.

5 At low levels of signal strength, small increases in signal strength will generally not suffice to make signal available. At high levels of signal strength, small increases in signal strength will generally not further improve availability. One would thus generally expect a sigmoid relationship between signal strength and the outcome of interest or signal strength and media availability (Adena et al. 2015, 1906-1908; Olken 2009, 25-28). 
some of the variation in signal strength leads to variation in media availability. Importantly, this approach can still be used-at the cost of extrapolation beyond the sample-if media availability is observed only for a subset of geographic units.

Finally, the third approach transforms signal strength into a binary indicator of media availability. Doing so requires information about what level of signal strength is sufficient for reception based on the technical characteristics and locations of receivers. We do not recommend this approach since the required information is typically unavailable. Moreover, the quality of radio or television reception varies continuously over much of signal strength's range, so that it is difficult to define a meaningful cutoff value below which reception is impossible and above which reception is feasible. While the technical literature and legal regulations suggest several cutoff values for "usable" signal, none of these cutoffs cleanly differentiates between areas or locations with and without media access. According to 47 C.F.R. $\S 73$, the cutoff level for television signal is $47 \mathrm{~dB} \mu \mathrm{V} / \mathrm{m}$. This cutoff level, which is calculated for rural areas, increases to approximately $60 \mathrm{~dB} \mu \mathrm{V} / \mathrm{m}$ in cities and $70 \mathrm{~dB} \mu \mathrm{V} / \mathrm{m}$ in major urban areas (International Telecommunications Union 2008). When receivers are outside buildings, or in the absence of noise or interference, the cutoff levels for cities and major urban areas are roughly the same as in rural areas (Haslett 2008). The cutoff values for $\mathrm{AM}$ and FM radio are similar for mono reception but about $6 \mathrm{~dB} \mu \mathrm{V} / \mathrm{m}$ higher for stereo reception (Ofcom 2010). When using an indicator variable for media availability we strongly recommend to report a range of estimates across plausible cutoff values (as in Crabtree et al. 2015). Moreover, if at all possible, cutoff values should be based on technical information about the capabilities of receivers in actual use (as in DellaVigna et al. 2014, 109-110). External information can sometimes be used to either pin down or at least bound the level of signal sufficient for reception. For example, Crabtree et al. (2015) use survey data and qualitative information to define a cutoff value for television availability that is known to be conservative and then show that using slightly higher cutoff values does not affect their results.

Finally, we should note that many works in the media effects literature in economics report effect sizes in terms of persuasion rates. These estimate the fraction of the audience of a media outlet that is convinced to change its behavior as a result of being exposed to the media outlet (see DellaVigna and Gentzkow 2010 for a formal definition). Since persuasion rates make it easier to compare effect sizes across studies, we recommend that political scientists report them in addition to more commonly reported quantities of interest.

\section{Causal Identification}

Existing research uses one of two identification strategies. When survey data on media exposure are available, signal strength can be used as an instrumental variable for media exposure (e.g., Olken 2009). In the absence of data on media exposure, variation in signal strength can be used to identify the intent-to-treat effect of mass media (e.g., Yanagizawa-Drott 2014; Crabtree et al. 2015). ${ }^{6}$

Both identification strategies rely on the insight that signal strength, in contrast to media exposure, is often plausibly exogenous since transmitters are located abroad (Crabtree et al. 2015) or have been installed by a previous regime (Enikolopov et al. 2011) or owner (Durante et al. 2017). Nevertheless, there are several ways in which the plausibility of the exogeneity assumption can be strengthened.

First, it is advantageous to control for "free-space" signal strength (Olken 2009, 26), the signal strength that would obtain in a given area or location if there were a direct line of sight between the transmitter and the receiver. By controlling flexibly for free-space signal strength researchers can isolate the variation in signal strength that is due only to topographical idiosyncrasies and the curvature of the Earth (Olken 2009). One thereby avoids identifying media effects off of

6 As helpfully pointed out by the anonymous reviewer, this is the relevant parameter from a policy perspective as only media availability (and not media exposure) can be manipulated. 
the distance from and technical characteristics of the transmitter, which could be endogenous (Durante et al. 2017). This approach works best in mountainous or hilly terrain (e.g., Olken 2009). If controlling for free-space signal strength is not feasible we suggest that researchers at least flexibly control for the distance to the nearest transmitter (e.g., Yanagizawa-Drott 2014).

In addition to controlling for free-space signal strength, regional or municipality fixed effects can be used to identify media effects only from residual variation due to geographical obstacles within small geographic areas characterized by similar economic and political conditions (e.g., Crabtree et al. 2015; Durante et al. 2017). When panel data are available and signal strength varies over time, as in Adena et al.'s (2015) study of radio in Weimar Germany, unit and time fixed effects imply that the main identifying assumption is that changes in signal strength are uncorrelated with time-varying unobservable determinants of the outcome of interest. A panel data framework can also be used to exploit the differential introduction of a media source across time and space, as in Olken (2009).

Finally, some research also controls for terrain characteristics such as average elevation and ruggedness (e.g., Olken 2009; Yanagizawa-Drott 2014; Durante et al. 2017). By allowing topographical obstacles to radio signal propagation to have an independent effect, one no longer exploits the presence of such obstacles for causal identification but only their particular shape. As Durante et al. (2017) point out, this is arguably a milder identifying assumption. As with free-space signal strength, this approach requires sufficient variation in terrain characteristics.

Finally, it is customary in the literature to provide indirect evidence of conditional exogeneity. One way to illustrate the plausibility of the conditional exogeneity assumption is to conduct placebo tests for outcomes that should not be affected by media availability. For instance, Enikolopov et al. (2011) show that the signal strength of NTV, a Russian television station, in 1999 was unrelated to voting behavior in 1995, before NTV became widely available. Relatedly, it is sometimes possible to exploit changes in media content over time (e.g., Adena et al. 2015) or the presence of several media sources with different content (e.g., DellaVigna et al. 2014). Another way to establish the credibility of the conditional exogeneity assumption is to show that conditional on free-space signal, regional fixed effects, terrain features, and perhaps other covariates, residualized signal strength is unrelated to covariates plausibly predictive of the outcome (e.g., Crabtree et al. forthcoming). Sensitivity tests for the presence of unobserved confounders in the spirit of Altonji, Elder, and Taber (2005) also see regular use (e.g., DellaVigna et al. 2014).

\section{An Applied Example}

To illustrate how electromagnetic signal propagation models can help researchers answer substantive questions we turn to the 2012 coup in Mali. ${ }^{7}$ On March 21, 2012, Malian military officers captured the Office of Radio and Television of Mali (ORTM). The junta fought hard to maintain control over the radio transmitters since it viewed radio as a vital means of projecting power and swaying public opinion.

Was control over ORTM actually beneficial to the military? In a recent article, Bleck and Michelitch $(2017,873)$ find that exposure to putschist-controlled radio "boosted national identity importance and willingness to delay elections." The authors support this claim with evidence from a field experiment of a radio distribution program that occurred across ten villages. In the absence of this field experiment, which by chance coincided with the coup, we might want to use an observational study to identify and estimate the causal effects of control over ORTM radio in Bamako, Mali's capital, during the military coup. We assume that we observe relevant outcome variables such as geocoded counter-coup protest events or individual-level public opinion survey data. We also assume that conditional on observables variation in radio

7 See Kern and Crabtree (2018) for replication materials. 


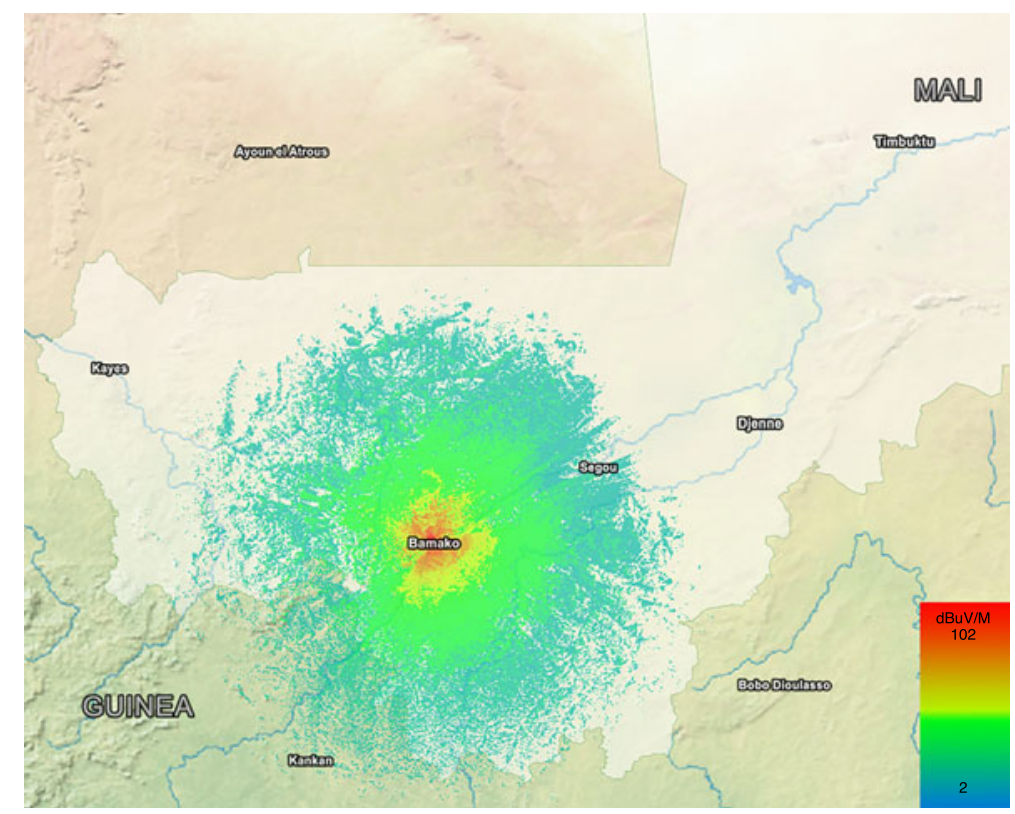

Figure 1. ORTM signal strength.

Table 2. Bamako's ORTM transmitters.

$\begin{array}{ccccccc}\text { Latitude } & \text { Longitude } & \text { ERP } & \text { Frequency } & \text { Height } & \text { Polarization } & \text { Direction } \\ 12.687211 & -8.025844 & 10 \mathrm{~kW} & 92.0 \mathrm{MHz} & 150 \mathrm{~m} & \text { horizontal } & \text { omnidirectional } \\ 12.687211 & -8.025844 & 10 \mathrm{~kW} & 95.2 \mathrm{MHz} & 150 \mathrm{~m} & \text { horizontal } & \text { omnidirectional }\end{array}$

Source: fmlist.org.

signal strength is exogenous so that the intent-to-treat effect of radio availability is identified. In practice this assumption would need to be defended, of course. Here, we focus on using ITM and publicly available data on ORTM transmitters in Bamako to create a signal strength measure that could serve as the treatment variable in an observational study (Table 2). Figure 1 plots the results. The multicolored area around Bamako represents the modeled coverage area for the ORTM transmitters located in the city; warmer colors are associated with stronger signal and cooler colors are associated with weaker signal. Without further information about the technical characteristics of radios in Mali, it is difficult to know exactly which areas received usable signal and which did not. We would thus flexibly include the continuous signal strength measure in an intent-to-treat outcomes model. While the specific estimation strategy would depend on available covariates and the precise identification assumptions, the general research design would rest on comparing units (e.g., areas, individuals) with different levels of signal strength after parametric or nonparametric adjustment for confounders.

\section{Conclusion}

Our hope is that this note further encourages social scientists to develop research designs that make use of naturally occurring variation in television and radio signal strength as a source of causal identification.

\section{Supplementary material}

For supplementary material accompanying this paper, please visit https://doi.org/10.1017/pan.2018.8. 


\section{References}

Adena, Maja, Ruben Enikolopov, Maria Petrova, Veronica Santarosa, and Ekaterina Zhuravskaya. 2015. Radio and the rise of the Nazis in prewar Germany. Quarterly Journal of Economics 130(4):1885-1939.

Altonji, Joseph G., Todd E. Elder, and Christopher R. Taber. 2005. Selection on observed and unobserved variables: Assessing the effectiveness of Catholic schools. Journal of Political Economy 113(1): 151-184.

Bleck, Jaimie, and Kristin Michelitch. 2017. Capturing the airways, capturing the nation? A field experiment on state-run media effects in the wake of a coup. Journal of Politics 79(3):873-889.

Crabtree, Charles, David Darmofal, and Holger L. Kern. 2015. A spatial analysis of the impact of West German television on protest mobilization during the East German revolution. Journal of Peace Research 52(3):269-284.

Crabtree, Charles, Holger L. Kern, and Steven Pfaff. Forthcoming. Mass media and the diffusion of collective action in authoritarian regimes: The June 1953 East German uprising. International Studies Quarterly.

DeBolt, Robert. n.d. Communication system planning tool: Full menu toolbar user guide. Typescript.

DellaVigna, Stefano, and Matthew Gentzkow. 2010. Persuasion: Empirical evidence. Annual Review of Economics 2: 643-669.

DellaVigna, Stefano, Ruben Enikolopov, Vera Mironova, Maria Petrova, and Ekaterina Zhuravskaya. 2014. Cross-border media and nationalism: Evidence from Serbian radio in Croatia. American Economic Journal: Applied Economics 6(3):103-132.

Durante, Ruben, Paolo Pinotti, and Andrea Tesei. 2017. The political legacy of entertainment tv. Working Paper.

Enikolopov, Ruben, Maria Petrova, and Ekaterina Zhuravskaya. 2011. Media and political persuasion: Evidence from Russia. American Economic Review 101(7):3253-3285.

Eppink, D., and W. Kuebler. 1994. TIREM/SEM handbook. Electromagnetic Compatibility Analysis Center Report ECAC-HDBK-93-076.

Haslett, Christopher. 2008. Essentials of radio signal propagation. Cambridge: Cambridge University Press.

Hufford, G. A., A. G. Longley, and W. A. Kissick. 1982. A guide to the use of the ITS Irregular Terrain Model in the area prediction mode. United States Department of Commerce, NTIA Report 82-100.

International Telecommunications Union. 2008. Recommendation ITU-R BS.412-9.

Jones, Graham A., David H. Layer, and Thomas G. Osenkowsky. 2013. National Association of Broadcasters engineering handbook. Burlington, MA: Taylor \& Francis.

Kern, Holger, and Charles Crabtree. 2018. Replication Data for: Using electromagnetic signal propagation models for radio and television broadcasts: An introduction. doi:10.7910/DVN/OZ6VYD, Harvard Dataverse, V1, UNF:6:f0HfB8C3VYDm1iUWrSZiiw==.

Lazaridis, Pavlos, Aristotelis Bizopoulos, Stylianos Kasampalis, John Cosmas, and Zaharias D. Zaharis. 2013. Evaluation of prediction accuracy for the Longley-Rice model in the FM and TV bands. XI International Conference - ETAI 2013, 26-28 September 2013, Lake Ohrid, FYR of Macedonia.

Longley, Anita G., and Patricia L. Rice. 1968. Prediction of tropospheric radio transmission loss over irregular terrain: A computer method. No. ITS-67. Boulder, CO: Institute for Telecommunication Sciences.

National Institute of Standards \& Technology (NIST). 2004. Significant papers from the first 50 years of the Boulder labs. Typescript.

Ofcom. 2010. Prediction of the 'useable' coverage of FM radio services. 2305/FMC/R/1/2.0.

Olken, Benjamin A. 2009. Do TV and radio destroy social capital? Evidence from Indonesian villages. American Economic Journal: Applied Economics 1(4):1-33.

Peisakhin, Leonid, and Arturas Rozenas. 2017. Electoral effects of biased media: Russian television in Ukraine. Working Paper.

Seybold, John S. 2005. Introduction to RF propagation. Hoboken, NJ: John Wiley \& Sons.

Yanagizawa-Drott, David. 2014. Propaganda and conflict: Evidence from the Rwandan genocide. Quarterly Journal of Economics 129(4):1947-1994. 